\title{
Circadian Rhythm, Energy Metabolism, and the Gut Microbiome: A Review
}

\author{
Bailey Vergara ${ }^{1}$ and Leya Joykutty ${ }^{1}$ \\ ${ }^{1}$ American Heritage School, Plantation, FL, USA \\ Advisor"
}

\section{ABSTRACT}

This review discussed the interrelationship between the circadian rhythm of the host and of the intestinal microbiome and the implications of this new field on energy metabolism, specifically implications relating to obesity. Circadian rhythm, or the 24-hour system that bodies use to expect and to increase receptivity to certain factors in the environment, is present in almost every organ and system. However, the data that this review utilized relates mainly to the intestinal microbiome: a group of bacteria, yeasts, and viruses that inhabit the colon, with this review's focus placed on bacteria. This interrelationship is mainly discussed through possible effects of circadian disruption and through an analysis of different methods by which the circadian rhythm of the host and the rhythm of the microbiome interact. TRF, or time-restricted feeding, has been identified as one of the potential methods to reduce the negative consequences of circadian dysregulation on metabolic processes, and various related studies were discussed in-depth. Data from four databases were analyzed to produce a variety of both experimental and review papers. All papers that related to the topic under review were thoroughly analyzed and incorporated into the review.

\section{Introduction}

This paper will examine the interrelationship between a host organism's circadian rhythm and the circadian rhythm of the intestinal microbiota and how they affect the energy metabolism of the host. Circadian dysregulation has been suggested as a contributing factor in the development of obesity and metabolic disorders in humans and mice, and these physiological consequences and their triggers will be explored. Examining the relationship between circadian rhythms, the gut microbiome, and energy metabolism may bring the scientific community closer to identifying a potential modifiable pathway to reducing risk of obesity.

Circadian rhythm is the body's natural, fluctuating rhythm based on a 24-hour day. Circadian rhythm regulates the oscillation of the expression of certain genes and the relative abundance of specific types of microorganisms, as well as the timing of certain biological processes according to the 24-hour clock. This clock is broken down into a variety of components, most notably the feed/fast cycle, the sleep/wake cycle, and the light/dark cycle. (R.M. Voigt et al, 2016, Christoph A. Thaiss, et al, 2014). Circadian rhythm in humans is managed by the central clock in the suprachiasmatic nucleus (SCN), located in the hypothalamus, which uses transcription factors CLOCK and Bmal1 and repressors such as Per and Cry to regulate the expression of certain genes (R.M. Voigt et al, 2016, Christoph A. Thaiss, et al, 2014). The circadian rhythm of the gut microbiome is a little harder to measure, but it is done so by tracking the fluctuations of different phyla (Amir Zarrinpar, et al, 2014). Microbial metabolites of the intestinal microbiome, which fluctuate according to these same cues, may also help the intestinal microbiome communicate with peripheral clocks (Xue Liang, et al, 2017). Individual microbes may also have their own rhythms, made up of three proteins that function similarly to those of the mammalian rhythms, as studied in Cyanobacteria (R.M. Voigt, et al, 2016). In addition to the clocks of the brain and the microbiome, there are a variety of smaller clocks, called peripheral clocks, located in different organs of the body. The central mammalian clock is mainly entrained by the host's light/dark schedule, while the peripheral clocks are entrained by both the central clock and host behaviors such as 
feeding (Joseph Bass, et al, 2010). When desynchrony, such as in the case of jet lag or shift work, is forced upon humans, natural circadian rhythm is disrupted and fluctuations of certain genes are impacted,which may lead to metabolic consequences (Simon N. Archer, et al, 2014, Margriet S. Westerterp-Plantenga, 2016).

Circadian rhythm and metabolism are deeply intertwined, especially when referring to the body's feed/fast cycles. One study has shown that meals eaten in the morning are more likely to be metabolized faster than those eaten in the evening (S. Bo, et al, 2015), and another study has proven that eating more calories during the day can lead to increased weight loss, as well as reduction of the levels of the appetite stimulant, ghrelin, in the blood (Daniela Jakubowicz, et al, 2013). However, it has also been shown that concentrations of blood variables may not fluctuate in accordance with circadian rhythm; instead, they may fluctuate based on meal patterns. Circadian disruption as a result of a phase-delay was shown to have no effect on energy expenditure (Hanne KJ Gonnissen, et al, 2012). In addition to meal patterns, sleep-related ailments have possibly detrimental effects on the body, with jet lag shown to reduce fluctuations of circadian genes, and sleep fragmentation possibly contributing to gut dysbiosis (Brittany A. Matenchuk, et al, 2020).

This review will look at the current research regarding circadian rhythm, the intestinal microbiome, and energy metabolism. It will explore the mechanisms by which the circadian clocks of the intestinal microbiome and the host can communicate, factors that disrupt circadian rhythms and how to reduce their effects, and the importance of daily fluctuations of microbiome composition to a healthy energy metabolism.

\section{Microbiome Compositions and Obesogenic Phenotypes}

The gut microbiome is composed of a variety of different organisms, including bacteria, yeasts, and viruses (Emanuele Rinninella, et al, 2019). This review will focus entirely on bacteria, including Firmicutes and Bacteroidetes, which make up $90 \%$ of the gut microbiome. Other phyla, which can be found in smaller numbers, include Fusobacteria, Actinobacteria, Proteobacteria, and Verrucomicrobia (Emanuele Rinninella, et al, 2019).

Bacteroides, of the phylum Bacteroidetes, the most abundant genus in the gut microbiome, is responsible for breaking down carbohydrates and the secretion of short-chain fatty acids, while bacteria of the phylum Firmicutes are responsible for the transport of certain nutrients, as well as the secretion of the short-chain fatty acid butyrate, while Verrucomicrobia promotes metabolic health (Shanthi G Parkar, et al, 2019). These three phyla are usually taken into account when discussing obesity and the microbiome, as Bacteroidetes and Verrucomicrobia levels have been shown to rise during fasting and fall during feeding, while Firmicutes fluctuate inversely (Amir Zarrinpar, et al, 2014). This may be related to the fact that high levels of Firmicutes and low levels of Bacteroidetes are associated with an obese microbiome (Amir Zarrinpar, et al, 2014). Shifts in circadian rhythms, specifically sleep/wake cycle shifts, have been linked to these increases and decreases, meaning that those with shifted sleeping patterns may be at a higher risk of obesity (Zhi Liu, et al, 2020). These increases and decreases are measured through the use of OTUs, or operational taxonomic units, which are ways to classify clusters of closely related microorganisms (Christoph A Thaiss, et al, 2016). Circadian rhythm disruptions, such as jet lag, have been shown to alter the diurnal fluctuations of OTUs, causing them to lose some of their rhythmicity, which can lead to an obesogenic microbiome (Christoph A. Thaiss, et al, 2014).

One study took another unique approach to examining the full extent of the impacts of the microbiome on the host and its circadian rhythms: effectively destroying the microbiome. When the microbiome was severely disrupted through antibiotics, the genes that began exhibiting circadian periodicity began to change. However, the behaviors of the host did not, meaning that the loss of meal patterns could not have been the reason for this reprogramming (Christoph A. Thaiss, et al, 2016). Several pathways lost and gained circadian rhythmicity in both the colon and the liver, including those linked to energy metabolism, broadening the understanding of the microbiome's effect on the body (Christoph A. Thaiss, et al, 2016) 


\section{Entrainment Agents}

Because the purpose of circadian rhythms is to train the body to anticipate certain things from its environment at certain times, it is only natural that these clocks must be entrained by external cues, such as light, feeding, and sleep.

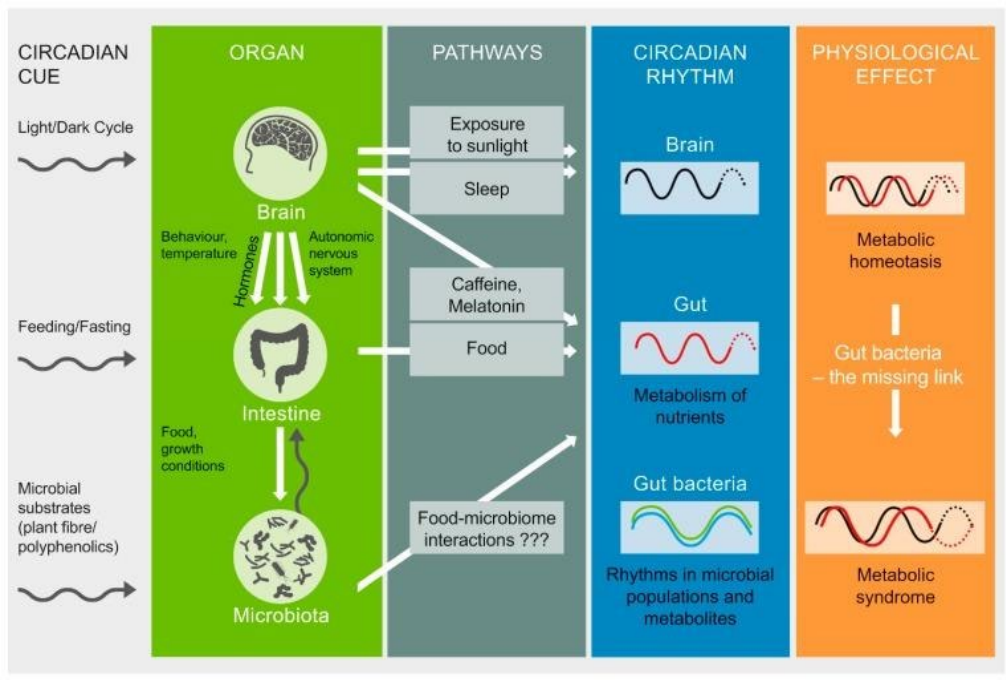

Fig 1- This figure shows the various entraining cues and pathways that lead to various physiological effects on the human body. Reprinted from Shanthi G Parkar, et al, 2019.

\section{Light/Dark Cycles}

Light/dark cycles determine the activity of most animals, as animals tend to restrict their activities to a certain portion of the cycle; for humans, activity is usually restricted to the light phase, meaning that feeding times are too. To that extent, the light/dark cycle also determines the fluctuations of certain phyla of bacteria. Restricting the feeding of mice to the active dark phase promotes the cyclical nature of the mouse microbiome. In mice, bacteria in the phylum Firmicutes and those in the phylums Bacteroidetes and Verrucomicrobia fluctuate inversely when feeding is limited to the dark phase (Shanthi G. Parkar, et al, 2019). The light/dark cycle is also the most potent entrainment agent for the SCN (Shanthi G Parkar, et al, 2019). Constant light exposure has been shown to increase and decrease levels of certain bacteria which may have roles in the protection and maintenance of the intestinal barrier (Jessica A Deaver, et al, 2018).

\section{Time-Restricted Feeding}

Time-restricted feeding — restricting feeding to an organism's active phase - is being explored as a potential solution to circadian disruption-related obesity. Most experiments that test TRF are conducted in mice, where promising results have emerged. In an experiment conducted in three groups of mice, mice that underwent time restricted feeding while still eating a high-fat, high-sugar diet maintained a relatively healthy microbiome composition (Amir Zarrinpar, et al, 2014). This may suggest that the benefits of time-restricted feeding could be due, at least in part, to alterations of the intestinal microbiome. Other findings from this study suggest that time-restricted feeding may increase calorie excretion in stool by restricting the microbiome's ability to break down complex sugars, contributing to weight loss. Another study conducted in mice used fecal transplantation to show that normalized feed/fast (and sleep/wake) cycles 
have the ability to restore fluctuations in the microbiome (Christoph A. Thaiss, et al, 2014). TRF may also restore the fluctuations of metabolic pathways, decrease appetite, and improve motor coordination (Amir Zarrinpar, et al, 2016). Some similar patterns have been found in human trials, where it has been shown that eating meals earlier in the day decreases the number of calories extracted from them. This may also contribute to decreases in appetite (Daniela Jakubowicz, et al, 2013).

The host organism's feeding patterns are not the only determinant for fluctuations in the composition of the gut microbiome. A study done in 2015 has shown that the gut microbiome undergoes fluctuations regardless of whether or not nutrients are absorbed through the gastrointestinal tract. Mice were only given nutrients through intravenous fluids (parenteral nutrition), which altered the composition of the microbiome, but still maintained cyclic variations (Vanessa Leone, et al, 2015).

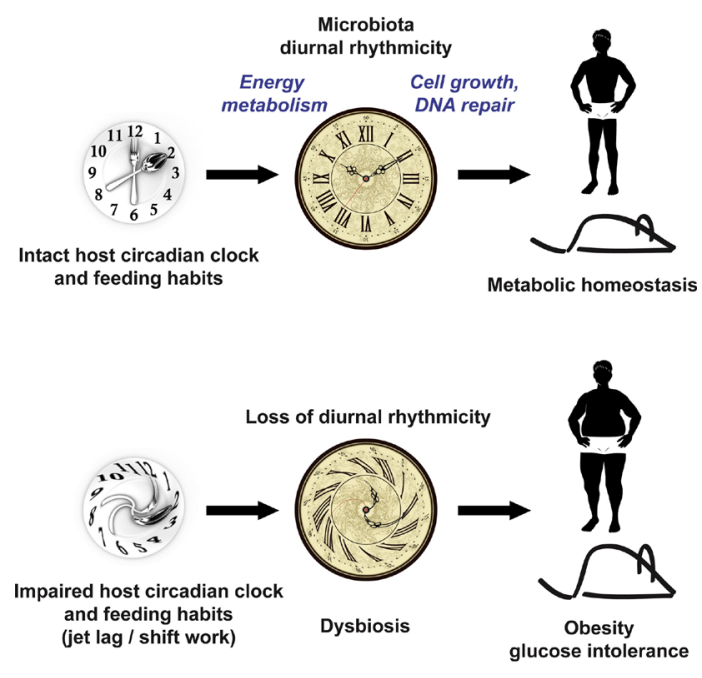

Fig 2- This figure shows the relationship between time-restricted feeding and obesity. Adapted from Graphical Abstract, Christoph A Thaiss, et al, 2014.

\section{Shift Work, Jet Lag, and Circadian Rhythms}

Shift work requires a worker to be awake for extended periods of time, usually interfering with natural sleep/wake and feed/fast patterns. This has devastating effects on energy metabolism, as it disrupts circadian cycles and uncouples the rhythms of systems that usually work in conjunction, shifting circadian rhythms. Shift work also desynchronizes the feed/fast cycle; workers have to eat at night to maintain energy, disrupting circadian rhythms (Amir Zarrinpar, et al, 2016). Shift workers experience decreased levels of leptin, increasing appetite, which may explain the link between shift work and obesity (Amir Zarrinpar, et al, 2016). Shift work has also been linked to type 2 diabetes and changes in genes related to sleep homeostasis, circadian rhythms, and metabolism (Möller-Levet, et al, 2013, Shan Z, et al, 2018). Jet lag may also predispose the organism to obesity through alterations in microbiome composition (Christoph A. Thaiss, 2018). Jet-lagged humans' fecal samples contain higher levels of bacteria associated with obesity, and, upon transplantation into rats, caused the rats to gain weight (Christoph A. Thaiss, et al, 2014).

\section{Potential Mechanisms of Communication and Their Effects on Circadian Rhythms and Energy Metabolism}


The intestinal microbiome releases a variety of metabolites, which are instrumental in communication between the intestinal microbiome and certain peripheral clocks. The body also releases hormones that fluctuate according to circadian rhythms, and these hormones, such as ghrelin and leptin, influence factors of energy metabolism and can be explored as a pathway for communication between the peripheral clocks and the SCN (Margriet S. WesterterpPlantenga, 2016).

\section{Ghrelin, Leptin, and Glucagon-Like Peptides}

Leptin is a hormone, secreted by adipose tissue, whose secretions exhibit circadian fluctuations, and it is in charge of the suppression of appetite (Margriet S. Westerterp-Plantenga, 2016, B Racz, et al, 2018). Levels are highest when appetite is lowest (usually at night) and lowest when appetite peaks during the day (Margriet S. Westerterp-Plantenga, 2016). BMI has been found to be associated with levels of leptin in blood plasma; essentially, the leptin is telling the body that it should stop eating, which makes leptin a factor in weight management (B Racz, et al, 2018). Leptin's counterpart, ghrelin, an appetite stimulant, is also a factor in weight management through the gut-brain axis (Margriet S. Westerterp-Plantenga, 2016, B Racz, et al, 2018). Ghrelin levels increase with the disruption of sleep cycles, making sleep-deprived people hungrier after they eat meals, which, in turn, may perpetuate obesity (Margriet S. WesterterpPlantenga, 2016, B Racz, et al, 2018). Ghrelin may also be used as a way for peripheral clocks to communicate with the SCN (Margriet S. Westerterp-Plantenga, 2016).

GLP-1, or Glucagon-Like Peptide-1, is a hormone secreted by intestinal cells that stimulates the secretion of insulin (Manuel Gil-Lozano, et al, 2014). Levels of GLP-1 can be used as a marker for decreased satiety, as levels shift with meal patterns (Margriet S. Westerterp-Plantenga, 2016). It, along with other hormones like ghrelin, can also act as feeding entrainment agents for the central nervous system and other tissues (Amir Zarrinpar, et al, 2016).

\section{Polyamines}

Polyamines are polycations that are being explored as a potential modifiable pathway to normalize circadian periods due to their roles in gene transcription and translation (Amir Zarrinpar, et al, 2016). The intestinal microbiota has been linked to the rhythmicity of these polyamines, and the absence of the gut microbiota has been shown to eliminate fluctuations in polyamines and other metabolites in mice (Christoph A Thaiss, et al, 2016). A diet lacking in polyamines has also been linked to the reprogramming of the transcriptome of hepatic cells in mice, which suggest that diet and its relationship with the microbiome have considerable effects on the circadian transcriptome of the liver (Christoph A Thaiss, et al, 2016). Polyamines have also been shown to affect the activities of clock proteins, thus helping in the maintenance of circadian rhythms (Amir Zarrinpar, et al, 2016). Polyamine levels normally decrease with age, leading to the lengthening of circadian periods, which normally last approximately 24 hours (Amir Zarrinpar, et al, 2016). Naturally, supplementation of these polycations may lead to the reversal of these lengthened circadian periods (Amir Zarrinpar, et al, 2016).

\section{Short-Chain Fatty Acids}

Short-chain fatty acids (SCFAs), which include acetate, propionate, and butyrate, are bacterial metabolites that may be involved in communication between the gut microbiome and peripheral clocks. Butyrate has demonstrated significant potential for altering circadian rhythms due to its ability to inhibit SIRT-1 (sirtuin-1), which plays a critical role in the maintenance of normalized circadian periods (Shanthi G Parkar, et al, 2019). Acetate has been shown to promote an obesogenic phenotype in some mice, and the effects of acetate and butyrate have also been shown in the modulation of the expression of certain circadian clock genes (Shanthi G Parkar, et al, 2019). The microbiomes of mice fed a high-fat diet have been shown to experience increased acetate production (Rachel J. Perry, et al, 2016). The presence 
of certain elements in one's diet, such as cellobiose, which facilitates the levels of certain SCFAs, may even contribute to peripheral clock re-entrainment (Yu Tahara, et al, 2018).

\section{Bile Acids}

Bile acids (BAs) are sent to the gastrointestinal tract to expedite the breakdown of lipids (Amir Zarrinpar, et al, 2016). While most bile acids are reabsorbed by the small intestines to the liver, some arrive at the colon, where they are metabolized by the bacteria who reside there. The microflora produces an enzyme which breaks down the bile salts into amino acids (Gucin Alp Avci, 2014). This process has been shown to regulate circadian genes such as Perl/2 and the clock-controlled genes Abcg5/8, Angptl4, and PPARv (Shanthi G. Parkar, et al, 2019). Bile acids can also be deconjugated to form unconjugated bile acids, which can undergo even more modifications and become secondary bile acids (Govindarajan, et al, 2016). It has been shown that unconjugated bile acids may increase the expression of the circadian genes that were associated with their conjugated counterparts (Govindarajan, et al, 2016). The cycling of bile acids not only regulates the expression of circadian genes, but also expresses a clear circadian rhythm (Shanthi G. Parkar, et al, 2019).

\section{Bacteria and Energy Harvesting}

The flora of the gut microbiome affect weight through energy harvesting. A $20 \%$ increase in bacteria from the phylum Firmicutes has been associated with an increased rate of calorie absorption: about 150 more calories a day (Reiner Jumpertz, et al, 2011). This same study also offers explanations for changes in the gut microbiome due to weight loss, particularly in the phyla Firmicutes and Bacteroidetes. The results of the experiment supported the hypothesis that changes in the composition of the intestinal microbiome may be due to the reduction of nutrients available in the host's diet rather than actual weight loss, with the microbiome sensing a shift in the nutrient load and changing the rate of nutrient absorption accordingly (Reiner Jumpertz, et al, 2011). This may indicate that the intestinal microorganisms may have a direct role in weight gain and weight loss; however, more research is needed to fully support this claim.

\section{Methodology}

Four databases were utilized in this search: PubMed, Journal PLOS, Nature, and the Cochrane Library.

Enter the key terms "circadian rhythm" "gut microbiome" in the search engine.

Use filter options to limit the years of the papers' publications to 2010-2020.

Repeat steps 2 and 3, this time using the terms "circadian rhythm" "energy metabolism".

Utilize applicable articles from references provided by articles returned from this search method to provide additional relevant information.

\section{Discussion}

The presented research appears to establish a clear connection between circadian rhythms of the host and microbiome, as well as their links to energy metabolism. However, there are still some connections left to be made. It has already been established that an increase in bacteria of the phylum Firmicutes and a decrease in Bacteroidetes are linked to weight gain and increased energy harvest (Amir Zarrinpar, et al, 2014, Reiner Jumpertz, et al, 2011); however, there must be some reason for these corresponding increases and decreases. Further understanding of the correlation between these phyla and obesity opens up many new pathways to decreasing a person's likelihood of becoming obese, 
possibly including supplementation of Bacteroidetes. Another area for future experiments to look into is the study of the molecular circadian clocks of individual microbes, as the circadian clock of Cyanobacteria may not be the same of those who inhabit human microbiomes (Emanuele Rinninella, et al, 2019, R.M. Voigt, et al, 2016).

Probiotics may be a promising pathway to minimize the effects of disruption on the microbial population, as promoting bacterial growth should be explored as a possible way to correct changes in altered operational taxonomic units (Amir Zarrinpar, et al, 2014, Christoph A Thaiss, et al, 2014). Probiotics may also indirectly affect microbiota composition, as probiotic supplementation can improve sleep and sleep quality (Brittany A. Matenchuk, et al, 2020). As research has previously shown, sleep regularity is an important factor in circadian rhythm synchronicity, and it may also affect microbiome health. Another potential pathway may be "postbiotics", the supplementation of microbial metabolites, which may help those with forced circadian disruption gain rhythmicity (Christoph A Thaiss, 2018). An example can be seen in the supplementation of polyamines to reverse the effects of declining polyamine levels of an individual's circadian rhythm (Amir Zarrinpar, et al, 2016). These avenues should be explored.

One significant gap in research in this field of study is that the majority of the studies that reference circadian rhythm and the gut microbiome are conducted in mice, not humans. While mice and humans have similar genetic makeup, there is diversity in humans that mice do not have, meaning that they are not an entirely accurate substitute for humans. Another thing that many human studies lack is a large sample size, which limits the generalizability of the study.

Many people suffer from the effects of circadian disruption because of jobs that demand it, and it is much easier and less ethically challenging to restrict a mouse's food intake than a human's. People have jobs that require them to be awake at night, and they still need to eat in order to function appropriately. Further research is needed in order to provide possible solutions and treatments for this group of workers, and as more evidence arises and more theories are supported, the scientific community will be able to bring them - and the world - one step closer to better management of weight gain and weight loss.

\section{Limitations}

These studies are an adequate basis for future research and conclusions. Qualitatively, they have proven a great help in assessing and analyzing the aspects of chronobiology covered in this review. Each paper comes to multiple conclusions, and most seem to support the hypotheses of one another, with many using each other as references for their papers. Quantitatively, however, the number of studies may not be sufficient to reach a definitive conclusion. These studies must be able to be replicated and reproduced to maximize scientific validity. Future studies may consider doing a meta-analysis, as there were a significant lack of these in the literature that was found, and increasing the sheer number of research collected may provide more grounds for future, more in-depth claims.

\section{Conclusion}

Body weight is an important factor in people's everyday lives, with effects ranging from health conditions to selfesteem. While people often rush to stores to partake in the latest weight loss fad, the real key to the maintenance of a healthy metabolic state may simply lie in understanding the rhythms of the body. Circadian rhythm and the intestinal microbiome are both implicated in the management of body weight. The likelihood of losing weight increases when meals are consumed earlier in the day (Daniela Jakubowicz, et al, 2013) and restricting feeding to certain times of day (TRF) may inhibit the microbiome's ability to break down complex carbohydrates into their more absorbable forms, increasing caloric loss in stool (Amir Zarrinpar, et al, 2014). Even important factors such as appetite, which is controlled in part by the hormones ghrelin and leptin, exhibit circadian periodicity (B Racz, et al, 2018). The body's receptivity to its environment is controlled by circadian rhythm, and when people don't follow their body's cues, 
harmful effects may occur, as seen in people who travel frequently and experience jet lag (Christoph A. Thaiss, 2018). If people really want to maintain a healthy energy metabolism, they need to simply listen to their body and follow their natural circadian rhythm.

\section{Acknowledgements}

I would like to thank Mrs. Leya Joykutty and Mr. Kepa Oyarbide for their help and support with this literature review. I truly could not have done this without them.

\section{References}

Alp Avc1, G. (2014). Bile Salts Deconjugation Using Microencapsulated Lactic Acid Bacteria Isolated from Handmade Yogurt. Journal of Food and Nutrition Research, 2(7), 340-343. https://doi.org/10.12691/jfnr-2-7-2

Archer, S. N., Laing, E. E., Möller-Levet, C. S., van der Veen, D. R., Bucca, G., Lazar, A. S., Santhi, N., Slak, A., Kabiljo, R., von Schantz, M., Smith, C. P., \& Dijk, D.-J. (2014). Mistimed sleep disrupts circadian regulation of the human transcriptome. Proceedings of the National Academy of Sciences, 111(6), E682-E691. https://doi.org/10.1073/pnas.1316335111

Bass, J., \& Takahashi, J. S. (2010). Circadian Integration of Metabolism and Energetics. Science, 330(6009), 13491354. https://doi.org/10.1126/science.1195027

Bo, S., Fadda, M., Castiglione, A., Ciccone, G., De Francesco, A., Fedele, D., Guggino, A., Parasiliti Caprino, M., Ferrara, S., Vezio Boggio, M., Mengozzi, G., Ghigo, E., Maccario, M., \& Broglio, F. (2015). Is the timing of caloric intake associated with variation in diet-induced thermogenesis and in the metabolic pattern? A randomized cross-over study. International Journal of Obesity, 39(12), 1689-1695. https://doi.org/10.1038/ijo.2015.138

Deaver, J. A., Eum, S. Y., \& Toborek, M. (2018). Circadian Disruption Changes Gut Microbiome Taxa and Functional Gene Composition. Frontiers in Microbiology, 9. https://doi.org/10.3389/fmicb.2018.00737

Gil-Lozano, M., Mingomataj, E. L., Wu, W. K., Ridout, S. A., \& Brubaker, P. L. (2014). Circadian Secretion of the Intestinal Hormone GLP-1 by the Rodent L Cell. Diabetes, 63(11), 3674-3685. https://doi.org/10.2337/db13-1501

Gonnissen, H. K., Rutters, F., Mazuy, C., Martens, E. A., Adam, T. C., \& Westerterp-Plantenga, M. S. (2012). Effect of a phase advance and phase delay of the 24-h cycle on energy metabolism, appetite, and related hormones. The American Journal of Clinical Nutrition, 96(4), 689-697. https://doi.org/10.3945/ajen.112.037192

Govindarajan, K., MacSharry, J., Casey, P. G., Shanahan, F., Joyce, S. A., \& Gahan, C. G. M. (2016). Unconjugated Bile Acids Influence Expression of Circadian Genes: A Potential Mechanism for Microbe-Host Crosstalk. PLOS ONE, 11(12), e0167319. https://doi.org/10.1371/journal.pone.0167319

Jakubowicz, D., Barnea, M., Wainstein, J., \& Froy, O. (2013). High Caloric intake at breakfast vs. dinner differentially influences weight loss of overweight and obese women. Obesity, 21(12), 2504-2512. https://doi.org/10.1002/oby.20460 
Jumpertz, R., Le, D. S., Turnbaugh, P. J., Trinidad, C., Bogardus, C., Gordon, J. I., \& Krakoff, J. (2011). Energybalance studies reveal associations between gut microbes, caloric load, and nutrient absorption in humans. The American Journal of Clinical Nutrition, 94(1), 58-65. https://doi.org/10.3945/ajen.110.010132

Leone, V., Gibbons, S., Martinez, K., Hutchison, A., Huang, E., Cham, C., Pierre, J., Heneghan, A., Nadimpalli, A., Hubert, N., Zale, E., Wang, Y., Huang, Y., Theriault, B., Dinner, A., Musch, M., Kudsk, K., Prendergast, B., Gilbert, J., \& Chang, E. (2015). Effects of Diurnal Variation of Gut Microbes and High-Fat Feeding on Host Circadian Clock Function and Metabolism. Cell Host \& Microbe, 17(5), 681-689. https://doi.org/10.1016/j.chom.2015.03.006

Liang, X., \& FitzGerald, G. A. (2017). Timing the Microbes: The Circadian Rhythm of the Gut Microbiome. Journal of Biological Rhythms, 32(6), 505-515. https://doi.org/10.1177/0748730417729066

Liu, Z., Wei, Z.-Y., Chen, J., Chen, K., Mao, X., Liu, Q., Sun, Y., Zhang, Z., Zhang, Y., Dan, Z., Tang, J., Qin, L., Chen, J.-H., \& Liu, X. (2020). Acute Sleep-Wake Cycle Shift Results in Community Alteration of Human Gut Microbiome. MSphere, 5(1). https://doi.org/10.1128/mSphere.00914-19

Matenchuk, B. A., Mandhane, P. J., \& Kozyrskyj, A. L. (2020). Sleep, circadian rhythm, and gut microbiota. Sleep Medicine Reviews, 53, 101340. https://doi.org/10.1016/j.smrv.2020.101340

Moller-Levet, C. S., Archer, S. N., Bucca, G., Laing, E. E., Slak, A., Kabiljo, R., Lo, J. C. Y., Santhi, N., von Schantz, M., Smith, C. P., \& Dijk, D.-J. (2013). Effects of insufficient sleep on circadian rhythmicity and expression amplitude of the human blood transcriptome. Proceedings of the National Academy of Sciences, 110(12), E1132-E1141. https://doi.org/10.1073/pnas.1217154110

Parkar, S., Kalsbeek, A., \& Cheeseman, J. (2019). Potential Role for the Gut Microbiota in Modulating Host Circadian Rhythms and Metabolic Health. Microorganisms, 7(2), 41. https://doi.org/10.3390/microorganisms 7020041

Perry, R. J., Peng, L., Barry, N. A., Cline, G. W., Zhang, D., Cardone, R. L., Petersen, K. F., Kibbey, R. G., Goodman, A. L., \& Shulman, G. I. (2016). Acetate mediates a microbiome-brain- $\beta$-cell axis to promote metabolic syndrome. Nature, 534(7606), 213-217. https://doi.org/10.1038/nature18309

RÁCZ, B., DUŠKOVÁ, M., STÁRKA, L., HAINER, V., \& KUNEŠOVÁ, M. (2018). Links Between the Circadian Rhythm, Obesity and the Microbiome. Physiological Research, S409-S420. https://doi.org/10.33549/physiolres.934020

Rinninella, E., Raoul, P., Cintoni, M., Franceschi, F., Miggiano, G., Gasbarrini, A., \& Mele, M. (2019). What is the Healthy Gut Microbiota Composition? A Changing Ecosystem across Age, Environment, Diet, and Diseases. Microorganisms, 7(1), 14. https://doi.org/10.3390/microorganisms7010014

Shan, Z., Li, Y., Zong, G., Guo, Y., Li, J., Manson, J. E., Hu, F. B., Willett, W. C., Schernhammer, E. S., \& Bhupathiraju, S. N. (2018). Rotating night shift work and adherence to unhealthy lifestyle in predicting risk of type 2 diabetes: Results from two large US cohorts of female nurses. BMJ, k4641. https://doi.org/10.1136/bmj.k4641

Society for Science and the Public (2019-20). International Science and Engineering Fair 2019-20: International Rules \& Guidelines. Washington, DC: Society for Science and the Public. 
Tahara, Y., Yamazaki, M., Sukigara, H., Motohashi, H., Sasaki, H., Miyakawa, H., Haraguchi, A., Ikeda, Y., Fukuda, S., \& Shibata, S. (2018). Gut Microbiota-Derived Short Chain Fatty Acids Induce Circadian Clock Entrainment in Mouse Peripheral Tissue. Scientific Reports, 8(1). https://doi.org/10.1038/s41598-018-19836-7

Thaiss, C., Zeevi, D., Levy, M., Zilberman-Schapira, G., Suez, J., Tengeler, A., Abramson, L., Katz, M., Korem, T., Zmora, N., Kuperman, Y., Biton, I., Gilad, S., Harmelin, A., Shapiro, H., Halpern, Z., Segal, E., \& Elinav, E. (2014). Transkingdom Control of Microbiota Diurnal Oscillations Promotes Metabolic Homeostasis. Cell, $159(3)$, 514-529. https://doi.org/10.1016/j.cell.2014.09.048

Thaiss, C. A. (2018). Microbiome dynamics in obesity. Science, 362(6417), 903-904. https://doi.org/10.1126/science.aav6870

Thaiss, C. A., Levy, M., Korem, T., Dohnalová, L., Shapiro, H., Jaitin, D. A., David, E., Winter, D. R., Gury-BenAri, M., Tatirovsky, E., Tuganbaev, T., Federici, S., Zmora, N., Zeevi, D., Dori-Bachash, M., Pevsner-Fischer, M., Kartvelishvily, E., Brandis, A., Harmelin, A., . . Elinav, E. (2016). Microbiota Diurnal Rhythmicity Programs Host Transcriptome Oscillations. Cell, 167(6), 1495-1510.e12. https://doi.org/10.1016/j.cell.2016.11.003

Voigt, R.M., Forsyth, C.B., Green, S.J., Engen, P.A., \& Keshavarzian, A. (2016). Circadian Rhythm and the Gut Microbiome. International Review of Neurobiology, 131, 193-205. https://doi.org/10.1016/bs.irn.2016.07.002

Westerterp-Plantenga, M. S. (2016). Sleep, circadian rhythm and body weight: Parallel developments. Proceedings of the Nutrition Society, 75(4), 431-439. https://doi.org/10.1017/S0029665116000227

Zarrinpar, A., Chaix, A., \& Panda, S. (2016). Daily Eating Patterns and Their Impact on Health and Disease. Trends in Endocrinology \& Metabolism, 27(2), 69-83. https://doi.org/10.1016/j.tem.2015.11.007

Zarrinpar, A., Chaix, A., Yooseph, S., \& Panda, S. (2014). Diet and Feeding Pattern Affect the Diurnal Dynamics of the Gut Microbiome. Cell Metabolism, 20(6), 1006-1017. https://doi.org/10.1016/j.cmet.2014.11.008 